CERN-TH/2001-272

\title{
UV/IR Mixing via Closed Strings and Tachyonic Instabilities
}

\author{
Adi Armoni ${ }^{\dagger}$ and Esperanza Lopez ${ }^{\ddagger}$ \\ $\dagger$ Theory Division, CERN \\ CH-1211 Geneva 23, Switzerland \\ adi.armoni@cern.ch \\ ‡ Max-Planck-Institut für Gravitationsphysik, Albert-Einstein-Institut, \\ Am Mühlenberg 1,D-14476 Golm, Germany \\ lopez@aei-potsdam.mpg.de
}

\begin{abstract}
We discuss UV/IR mixing effects in non-supersymmetric non- commutative $U(N)$ gauge theories. We show that the singular (nonplanar) terms in the 2- and 3-point functions, namely the poles and the logarithms, can be obtained from a manifestly gauge invariant effective action. The action, which involves open Wilson line operators, can be derived from closed strings exchange between two stacks of Dbranes. Our concrete example is type 0B string theory and the field theory that lives on a collection of $N$ electric D3-branes. We show that one of the closed string modes that couple to the field theory operator which is responsible for the infrared poles, is the type 0 tachyon.
\end{abstract}




\section{Introduction}

Non-commutative gauge theories attracted recently a lot of attention, mainly due to the discovery of their relation to string/M theory [1, 2]. The perturbative dynamics of these theories is very interesting: planar graphs of non-commutative theories are exactly the same as the planar graphs of ordinary theories apart from global phases which depend on external momenta [3, 4, 5]. Non-planar graphs, on the other hand, are regulated by the noncommutativity parameter $\theta$ and they are therefore UV-finite. This regularization is however only effective when there is a non-zero momentum inflow into the graph. In particular, as a result of this, the non-planar contribution to the propagator contains, usually, a pole $1 /(\theta p)^{2}$. This pole, which originates from the high momentum region of the integral (UV) seems to affect the large distance dynamics. This unusual phenomenon is called UV/IR mixing [6]. In supersymmetric theories this pole cancels and a softer version of UV/IR mixing exists due to a logarithmic contribution [7]. Aspects of the UV/IR mixing phenomena in scalar theories [8, 9, 10, 11, 12, 13] as well as gauge theories [14, 15, 16, 17, 18, 19, 20, 22, 21, 23, 24] were studied by many authors over the past two years. See [25, 26] for comprehensive reviews.

In this work we would like to focus on non-supersymmetric non-commutative gauge theories in 4-dimensions. The non-planar pole modifies the dispersion relation of the photon as follows [7, 22, 23]

$$
E^{2}=\vec{p}^{2}-\left(N_{B}^{a d j}-N_{F}^{a d j}\right) \frac{g^{2}}{\pi^{2}} \frac{1}{(\theta p)^{2}},
$$

where $N_{B}^{a d j}$ and $N_{F}^{a d j}$ are the numbers of bosons and fermions in the adjoint representation, respectively. In the case of pure Yang-Mills theory, or in general when $N_{B}^{a d j}>N_{F}^{a d j}$, the low momentum end of the spectrum acquires imaginary energy. Namely, the one loop analysis suggests that the theory suffers from an instability. In [22] it was shown that the quadratic pole-like infrared divergence is gauge-fixing-independent. In 23] non-commutative $\mathcal{N}=4$ Yang-Mills at finite temperature was considered. This theory presents a regularized version of UV/IR mixing, where the temperature acts as a UV cut-off. Although perturbation theory seems to be under better control in this case, tachyonic excitations at long wavelength appear for $T$ bigger than a critical temperature $T_{c} \sim 1 / \sqrt{g \theta}$. All this suggests that the associated instability might not be an artefact of perturbation theory, but instead its 
source should be searched in an expansion around the wrong vacuum. Of course, the existence of a stable vacuum is an open question.

As already mentioned, apart from the tachyonic poles in the non-planar graphs, these graphs contain also subleading logarithmic contributions. These contributions do not change the photon dispersion relation and are not expected to cause any instabilities, but they are relevant in the IR. In particular, they affect the effective coupling [20].

The purpose of this note is to gain a better understanding of the tachyonic poles and the logarithmic effects by using string theory. We summarize briefly our main results. We consider a field theory that lives on D3 electric branes of the non-supersymmetric type $0 \mathrm{~B}$ string theory. We show that all singular amplitudes involving pole-like infrared divergences can be encoded in a rather simple gauge-invariant effective action

$$
S_{e f f}^{I} \sim\left(N_{B}^{a d j}-N_{F}^{a d j}\right) \int \frac{d^{4} p}{(2 \pi)^{4}} \operatorname{tr} W(p) \operatorname{tr} W(-p) \frac{m^{2}}{\tilde{p}^{2}} K_{2}(m \tilde{p}),
$$

where $W(p)$ denotes the open Wilson loop operator [27, 28]. This effective action is structurally analogous to a closed string exchange between two stacks of D-branes. This coincidence is more than formal since we will see that the Bessel function kernel in (2) can be directly related to a closed string propagator in type 0 string theory. Although (2) has the form of a closed string exchange, all the tower of closed string modes contribute to it, similarly to the proposal by [8]. Among the closed string modes that couple to the open Wilson line operator is the type 0 closed string tachyon. This is in contrast to the ordinary commutative case, where the tachyon just contributes to the vacuum energy of the field theory. The fact that the closed string tachyon couples to a non-trivial operator in the field theory, which in addition is responsible of the pole-like infrared divergences, suggests that there could be a relation between string and non-commutative instabilities.

Although at a more intuitive level, our analysis can be extended to the logarithmic infrared-divergent terms. They admit the following gauge-invariant completion

$$
\begin{aligned}
S_{e f f}^{I I} \sim \beta_{0} \int \frac{d^{4} p}{(2 \pi)^{4}}\{ & \operatorname{tr} F_{\mu \nu} W(p) \operatorname{tr} F_{\mu \nu} W(-p)+ \\
& \left.\operatorname{tr} D_{\mu} \phi_{i} W(p) \operatorname{tr} D_{\mu} \phi_{i} W(-p)\right\} K_{0}(m \tilde{p}) .
\end{aligned}
$$


This expression is only schematic; a precise definition of $S_{e f f}^{I I}$ is presented in section 4. From a string point of view, we can interpret (3) as due to the exchange of massive 2-form closed strings. Note that this sector of the closed string does not contain tachyons. Indeed, as already mentioned, there is no tachyonic instability associated with the logarithmic part of the action.

The organization of this article is as follows: in section 2 we describe our model and we calculate the various singular amplitudes. Sections 3 is devoted to a derivation of the full gauge invariant effective action related to the infrared pole-like terms in the case of pure $U(1)$ theory. In section 4 we suggest a derivation of the effective action via closed strings exchange between D3-branes of type 0 string theory. We discuss our results in section 5 .

We use the following notations and conventions. The field theory under consideration is a $4 \mathrm{~d}$ one with space-space non-commutativity $\left[x^{1}, x^{2}\right]=i \theta$ (or a tensor $\theta^{\mu \nu}$ with non-vanishing components in the 1,2 directions). We also use the notation $\tilde{p}^{\mu}=\theta^{\mu \nu} p_{\nu}$. The $U(N)$ generators are normalized such that $\operatorname{tr} t^{A} t^{B}=\frac{1}{2} \delta^{A B}$ and in particular the $U(1)$ generator is $t^{0}=\frac{1}{\sqrt{2 N}}$.

Note added: as we finished our work, paper [29] appeared. The author of this paper arrived to the result (2) and discussed it from the matrix theory perspective.

\section{Field Theory Calculations - Various Non-Planar Amplitudes}

In this section we describe the UV/IR mixing effects in a concrete model. The $4 \mathrm{~d}$ field theory under consideration is the theory that lives on a stack of $N$ coincident electric D-branes of type 0B string theory. It is obtained by dimensional reduction of pure (non-supersymmetric) 10d non-commutative Yang-Mills theory. The model contains a vector and 6 adjoint scalars and it is described by the following action

$$
S=\operatorname{tr} \int d^{4} x\left(-\frac{1}{2 g^{2}} F_{\mu \nu} \star F^{\mu \nu}+D_{\mu} \phi^{i} \star D^{\mu} \phi^{i}\right)
$$

where,

$$
F_{\mu \nu}=\partial_{\mu} A_{\nu}-\partial_{\nu} A_{\mu}-i\left(A_{\mu} \star A_{\nu}-A_{\nu} \star A_{\mu}\right)
$$


and

$$
D_{\mu} \phi^{i}=\partial_{\mu} \phi^{i}-i\left(A_{\mu} \star \phi^{i}-\phi^{i} \star A_{\mu}\right)
$$

for $i=1$...6. The model is invariant under the following non-commutative $U(N)$ gauge transformation

$$
\begin{aligned}
& \delta_{\lambda} A_{\mu}=\partial_{\mu} \lambda-i\left(A_{\mu} \star \lambda-\lambda \star A_{\mu}\right) \\
& \delta_{\lambda} \phi^{i}=-i\left(\phi^{i} \star \lambda-\lambda \star \phi^{i}\right) .
\end{aligned}
$$

Let us focus on the one-loop structure of the theory. The planar sector is well understood. Apart from global phases associated to external legs, the various amplitudes are the same as in the commutative cousin of the theory [5]. In particular the theory is one-loop renormalizable with the same counterterms as those of the commutative theory [16.

The non-planar sector of the theory exhibits an interesting pattern. In this case the Moyal phases associated with the vertices do not cancel and lead to a UV-finite result [6]. Consider first the propagator of the gluon. The only non-vanishing non-planar graph exists when the external gluons are in the $U(1)$ [6, 16]. The sum of the various contribution, due to gluons, ghosts and scalars running in the loops yields [14, 0, 15, 16]

$$
A_{(1-1)}^{\mu \nu}=-8 g^{2} N \int \frac{d^{4} q}{(2 \pi)^{4}} \frac{\left(2 q^{\mu} q^{\nu}-g^{\mu \nu} q^{2}\right)}{q^{4}} \exp 2 i \tilde{p} q=\frac{64 g^{2} N}{(4 \pi)^{2}} \frac{\tilde{p}^{\mu} \tilde{p}^{\nu}}{\tilde{p}^{4}} \text {. }
$$

A similar calculation yields a similar result for the scalar propagator

$$
A_{(1-1)}=8 g^{2} N \int \frac{d^{4} q}{(2 \pi)^{4}} \frac{1}{q^{2}} \exp 2 i \tilde{p} q=\frac{32 g^{2} N}{(4 \pi)^{2}} \frac{1}{\tilde{p}^{2}} .
$$

Note that there is a relative factor of 2 between (9) and (10).

The poles in (9) and (10) signal the interesting UV/IR mixing that is typical of non-commutative theories. The origin of these contributions is the UV regime and they seem to affect the IR of the theory. These poles create a potential problem in the renormalization process, since when the non-planar graphs are inserted in higher loop diagrams they create new divergences. It was suggested [6] that, in certain cases, the sum of the geometric series of these contributions can shift the pole such that these new divergences are avoided. This procedure, however, cannot be implemented in the present case, due to the positive sign in front of (9) and (10) [22, 23]. In general, 
the coefficient in front of $(9)$ and (10) is determined by the number of bosons in the adjoint representation minus the number of fermions in the adjoint representation [23]. In cases where there are more bosons than fermions in the adjoint (such as the present case), a resummation of the series is impossible, since the series does not converge. At present, there is no known procedure to cure this pathology. In particular, the pure non-commutative Yang-Mills theory seems to be sick.

Let us now proceed to the non-planar corrections to the 3-point vertices. The pattern is similar: whereas planar graphs take the same form as in the commutative theory and they are divergent, non-planar graphs are UV-finite, but singular at $\theta \rightarrow 0$. Non-vanishing diagrams involve $U(1)$ factor in at least one of the external legs [16]. The amplitude in the case of 3 external gluons, when all gluons are in the $U(1)$ is

$$
A_{(1-1-1)}^{\mu \nu \rho}=\frac{i 64 g^{3} \sqrt{N / 2}}{(4 \pi)^{2}}\left(\frac{\tilde{p}_{1}^{\mu} \tilde{p}_{1}^{\nu} \tilde{p}_{1}^{\rho}}{\tilde{p}_{1}^{4}}+\frac{\tilde{p}_{2}^{\mu} \tilde{p}_{2}^{\nu} \tilde{p}_{2}^{\rho}}{\tilde{p}_{2}^{4}}+\frac{\tilde{p}_{3}^{\mu} \tilde{p}_{3}^{\nu} \tilde{p}_{3}^{\rho}}{\tilde{p}_{3}^{4}}\right) .
$$

We have ignored in this expression a factor $\cos \tilde{p}_{1} p_{2} / 2$, which appears in previous calculations of the leading IR contribution to the 3-point function. The reason is that, in the approximation used to obtain (11), i.e. $\tilde{p}_{i} p_{j} \ll 1$, we cannot distinguish between $\cos \tilde{p}_{1} p_{2} / 2$ and 1 . We keep this convention in the following. When one gluon is in the $U(1)$ and the two other gluons are in the $S U(N)$ the amplitude takes the form

$$
A_{(1-N-N)}^{\mu \nu \rho}=\frac{i 64 g^{3} \sqrt{N / 2}}{(4 \pi)^{2}} \frac{\tilde{p}_{1}^{\mu} \tilde{p}_{1}^{\nu} \tilde{p}_{1}^{\rho}}{\tilde{p}_{1}^{4}}
$$

where $\tilde{p}_{1}$ is the momentum of the $U(1)$ field. Similarly, the amplitude for two external scalars and one gluon, all in the $U(1)$, is

$$
A_{(1-1-1)}^{\mu}=\frac{i 32 g^{3} \sqrt{N / 2}}{(4 \pi)^{2}}\left(\frac{\tilde{p}_{1}^{\mu}}{\tilde{p}_{1}^{2}}+\frac{\tilde{p}_{2}^{\mu}}{\tilde{p}_{2}^{2}}+\frac{\tilde{p}_{3}^{\mu}}{\tilde{p}_{3}^{2}}\right) .
$$

In the case of two scalars and one gluon transforming in $U(1)$ and $S U(N)$ the amplitude is the following

$$
A_{(1-N-N)}^{\mu}=\frac{i 32 g^{3} \sqrt{N / 2}}{(4 \pi)^{2}} \frac{\tilde{p}_{1}^{\mu}}{\tilde{p}_{1}^{2}}
$$


where, again, $\tilde{p}_{1}$ is the momentum of the $U(1)$ field.

The information about the various non-planar diagrams can be summarized in the following effective action

$$
\begin{aligned}
& \frac{\pi^{2}}{2} S_{I}=g^{2} \int d^{4} p\left(2 \frac{\tilde{p}^{\mu} \tilde{p}^{\nu}}{\tilde{p}^{4}} \operatorname{tr} A_{\mu}(-p) \operatorname{tr} A_{\nu}(p)+\frac{1}{\tilde{p}^{2}} \operatorname{tr} \phi^{i}(-p) \operatorname{tr} \phi^{i}(p)\right) \\
& +\frac{i g^{3}}{(2 \pi)^{4}} \int d^{4} p_{1} d^{4} p_{2} d^{4} p_{3} \delta\left(p_{1}+p_{2}+p_{3}\right) \times \\
& \left\{2 \frac{\tilde{p}_{1}^{\mu} \tilde{p}_{1}^{\nu} \tilde{p}_{1}^{\rho}}{\tilde{p}_{1}^{4}} \operatorname{tr} A_{\mu}\left(p_{1}\right) \operatorname{tr} A_{\nu}\left(p_{2}\right) A_{\rho}\left(p_{3}\right)\right. \\
& \left.+\frac{\tilde{p}_{1}^{\mu}}{\tilde{p}_{1}^{2}}\left(\operatorname{tr} A_{\mu}\left(p_{1}\right) \operatorname{tr} \phi^{i}\left(p_{2}\right) \phi^{i}\left(p_{3}\right)+2 \operatorname{tr} \phi^{i}\left(p_{1}\right) \operatorname{tr} \phi^{i}\left(p_{2}\right) A^{\mu}\left(p_{3}\right)\right)\right\} .
\end{aligned}
$$

Apart from the terms which are summarized in the effective action (15), there are other contributions which are less singular when $\theta \rightarrow 0$. In contrast to the poles, these terms (which as we shall see in a moment are log-like terms) do not cancel even in the supersymmetric case, apart from the $\mathcal{N}=4 \mathrm{SYM}$ case [7]. These terms have a different Lorentz structure than the poles and they are all proportional to the one-loop beta function coefficient. Most of our analysis of this part is based on [18] and [20].

The gluon propagator (for the $U(1)$ degrees of freedom) contains the following non-planar contribution

$$
M_{(1-1)}^{\mu \nu}=-\frac{26 g^{2} N}{3(4 \pi)^{2}}\left(p^{2} g^{\mu \nu}-p^{\mu} p^{\nu}\right) \log m^{2} \tilde{p}^{2},
$$

where $m^{2}$ is an IR cut-off. We can think about it as a mass term for the scalars (and vectors), given via a Higgs mechanism. Similarly to the gluon, the correction to the scalar propagator is

$$
M_{(1-1)}=-\frac{26 g^{2} N}{3(4 \pi)^{2}} p^{2} \log m^{2} \tilde{p}^{2} .
$$

The subleading corrections to the 3-point vertices are the following: for 3 gluons, all in the $U(1)$, we have

$$
M_{(1-1-1)}^{\mu \nu \rho}=-\frac{i 26 g^{3} \sqrt{N / 2}}{3(4 \pi)^{2}} \sin \left(\frac{1}{2} \tilde{p}_{1} p_{2}\right)\left(\log m^{2} \tilde{p}_{1}^{2} g^{\nu \rho} p_{1}^{\mu}+\text { perm. }\right)
$$


where 'perm.' means permutations of the three momenta and Lorentz indices due to the symmetry of the amplitude. Similarly for the case of 1 gluon in the $U(1)$ and 2 gluons are in the $S U(N)$

$$
M_{(1-N-N)}^{\mu \nu \rho}=-\frac{i 26 g^{3} \sqrt{N / 2}}{3(4 \pi)^{2}} \sin \left(\frac{1}{2} \tilde{p}_{1} p_{2}\right)\left(\log m^{2} \tilde{p}_{1}^{2} g^{\nu \rho} p_{1}^{\mu}+\text { perm. }\right),
$$

where now $p_{1}$ is the momentum of the $U(1)$ gluon, and the permutations are with respect to the 2 gluons in the $S U(N)$.

In the case of amplitudes where there are two scalars and one gluon we have

$$
M_{(1-1-1)}^{\mu}=-\frac{i 26 g^{3} \sqrt{N / 2}}{3(4 \pi)^{2}} \sin \left(\frac{1}{2} \tilde{p}_{1} p_{2}\right)\left(\log m^{2} \tilde{p}_{1}^{2} p_{1}^{\mu}+\text { perm. }\right),
$$

and the same expression for the $S U(N)-S U(N)-U(1)$ amplitude.

The log-like amplitudes can be summarized by the following effective action

$$
\begin{aligned}
& -\frac{24 \pi^{2}}{13} S_{I I}=g^{2} \int d^{4} p\left(\left(p^{2} g^{\mu \nu}-p^{\mu} p^{\nu}\right) \log m^{2} \tilde{p}^{2}\left(\operatorname{tr} A^{\mu}(-p)\right)\left(\operatorname{tr} A^{\nu}(p)\right)\right. \\
& +p^{2} \log m^{2} \tilde{p}^{2}\left(\operatorname{tr} \phi^{i}(-p)\right)\left(\operatorname{tr} \phi^{i}(p)\right) \\
& +\frac{i g^{3}}{(2 \pi)^{4}} \int d^{4} p_{1} d^{4} p_{2} d^{4} p_{3} \delta\left(p_{1}+p_{2}+p_{3}\right) \times \sin \left(\frac{1}{2} \tilde{p}_{1} p_{2}\right) \times \log m^{2} \tilde{p}_{1}^{2} p_{1}^{\mu} \times \\
& \left\{\left(\operatorname{tr} A^{\nu}\left(p_{1}\right)\right)\left(\operatorname{tr} A^{\mu}\left(p_{2}\right) A^{\nu}\left(p_{3}\right)\right)+\left(\operatorname{tr} \phi^{i}\left(p_{1}\right)\right)\left(\operatorname{tr} A^{\mu}\left(p_{2}\right) \phi^{i}\left(p_{3}\right)\right)\right\} .
\end{aligned}
$$

The actions (15)(21) are not gauge invariant. In order to have a (noncommutative) gauge invariant action, higher order terms in $A_{\mu}$ should be added. In the following sections we will derive a manifestly gauge-invariant action which includes (15) and (21) as part of it.

\section{The Effective Action - Field Theory Derivation}

\subsection{The Poles}

We will start by considering the pole-like IR-divergent contributions to the

2- and 3-point functions with only gluons as external legs. We observe that 
in both cases each vector field $A_{\mu}\left(p_{i}\right)$ is contracted with $\tilde{p}^{\mu}=\theta^{\mu \nu} p_{\nu}$, where $p_{\nu}$ is the total momentum flowing on each trace operator. This suggests that these terms are related to the simplest gauge-invariant operators carrying non-zero momentum, the straight open Wilson line defined by [27, 28]

$$
W(p)=\operatorname{tr} \int d^{4} x P_{*}\left(e^{i g \int_{0}^{1} d \sigma \tilde{p}^{\mu} A_{\mu}(x+\tilde{p} \sigma)}\right) * e^{i p x} .
$$

Indeed, the gluon 2- and 3-point functions (15) can be obtained as the first terms in the expansion of the following gauge-invariant expression

$$
S_{e f f}^{I}=\frac{2+N_{s}}{2 \pi^{2}} \int d^{4} p W^{\prime}(-p) f(\tilde{p}) W^{\prime}(p),
$$

with $f(\tilde{p})$ a function that tends in the IR to $1 / \tilde{p}^{4} ; N_{s}$ is the number of scalars in the adjoint representation $\left(N_{s}=6\right.$ in the type 0 case). We denote by $W^{\prime}(p)$ the Wilson loop operator (22) once the $O\left(g^{0}\right)$ term has been subtracted

$$
W^{\prime}(p)=i g \tilde{p}^{\mu} \operatorname{tr} A_{\mu}(p)-g^{2} \int \frac{d^{4} l}{(2 \pi)^{4}} \frac{\sin \frac{\tilde{l} p}{2}}{\tilde{l} p} \tilde{p}^{\mu} \tilde{p}^{\nu} \operatorname{tr} A_{\mu}(p-l) A_{\nu}(l)+\ldots
$$

By inserting (24) in (23), we immediately recover the gluon 2-point function. The expressions (11),(12) for the gluon 3-point function are valid in the limit $\tilde{p}_{i} p_{j}<<1$. In that limit $\sin \frac{\tilde{l} p}{2} / \frac{\tilde{p} p}{2} \rightarrow 1$ and thus also the 3 -point function is correctly obtained from (23). This was to be expected since the IR divergent contribution to the 3-point function satisfies the Ward identity [18.

For the pure $U(1)$ non-commutative theory, (23) can be obtained from a direct calculation of the 1-loop N-point functions. This will allow us to determine the function $f$ in (23). Due to the structure of the argument in the exponential of the Wilson loop, (23) contributes to the N-point function with terms proportional to $\tilde{p}^{\mu_{1}} \ldots \tilde{p}^{\mu_{N}}$. The N-point functions will have in general a complicated Lorentz index structure. However it is easy to isolate the terms of the mentioned form. They can only come from diagrams with 3 -point vertices. Diagrams with 4 -point vertices will give rise to a tensor structure containing $g^{\mu_{i} \mu_{j}}$, and therefore are not of the desired form. We will like to point out that diagrams with 4-point vertices can produce as strong an IR divergence as those with only 3 -point vertices. Indeed, the tadpole induces a quadratic pole-like contribution to the 2-point function of the form $g^{\mu \nu} / \tilde{p}^{2}$. However the role of this term is to cancel a similar contribution coming from 


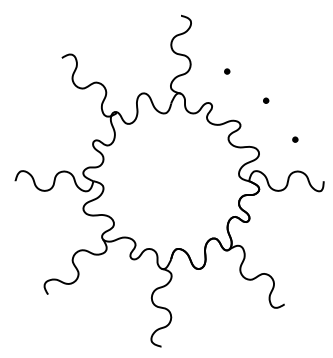

a

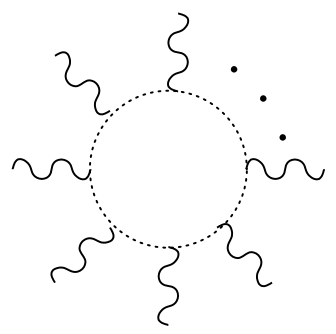

$\mathrm{b}$

Figure 1: Amplitudes containing terms $\sim \tilde{p}^{\mu_{1}} \ldots \tilde{p}^{\mu_{N}}$. Wavy lines refer to gauge bosons and doted lines to ghost. The end points of the external lines are background vector fields $B_{\mu}$.

diagrams with 3-point vertices, and which would otherwise violate the Ward identity [7]. The same applies to the 3-point function. We will thus ignore diagrams with 4-point vertices when analizing the leading UV/IR mixing effects.

We will use the background field method in the following analysis; for the associated Feynman rules see [18]. The diagrams we are interested in are those depicted in fig.1. We have

$$
\begin{aligned}
& (a)+(b)= \\
& (-2 i g)^{N} \int \frac{d^{4} l}{(2 \pi)^{4}} \prod_{i=1}^{N} \frac{\left(2 l+2 p_{1}+. .+2 p_{i-1}+p_{i}\right)_{\mu_{i}}}{\left(l+p_{1}+. .+p_{i-1}\right)^{2}} \sin \frac{\tilde{p}_{i}\left(l+p_{1}+. .+p_{i-1}\right)}{2} .
\end{aligned}
$$

As explained, we will disregard those parts of (25) whose tensor structure is such that they cannot contribute to (23). This allows us to discard all the terms in the numerator proportional to external momenta, and keep only $2 l_{\mu_{i}}$ for each $i$. Expression (25) then reduces to

$$
(-2 i g)^{N} \sum_{\nu_{i}}(-)^{n} \int \frac{d^{4} l}{(2 \pi)^{4}} \frac{l_{\mu_{1} . .} l_{\mu_{N}} e^{-i \tilde{p} l-\frac{i}{2} \sum_{j<k} \tilde{p}_{j} p_{k} \nu_{k}}}{l^{2}\left(l+p_{1}\right)^{2} \ldots\left(l+p_{1}+. .+p_{N-1}\right)^{2}},
$$

where the summation on $\nu_{i}, \nu_{i}= \pm 1$ for $i=1, . ., N$, comes from expanding the sine. We have defined $p=\sum_{i} p_{i} \frac{1-\nu_{i}}{2}$ and $n=\sum_{i} \frac{1-\nu_{i}}{2}$. We can interpret 
the $\mathrm{N}$ vertices as twisted or untwisted depending if $\nu=-1$ or 1 respectively. Thus $n$ is the number of twisted vertices and $p$ the total momentum flowing in the twisted vertices. The $l_{\mu_{i}}$ in the numerator can now be substituted by derivatives with respect to $\tilde{p}^{\mu}$ acting on the exponential. In order to simplify the analysis will we consider $\tilde{p}$ as an independent variable, and only in the end we will set $\tilde{p}^{\mu}=\theta^{\mu \nu} p_{\nu}$ with $p=\sum_{i} p_{i} \frac{1-\nu_{i}}{2}$. This allows us to bring the derivatives out of the integral, and rewrite (26) as

$$
(-2 i g)^{N} \partial_{\mu_{1}} \ldots \partial_{\mu_{N}} \sum_{\nu_{i}}(-)^{n} \int \frac{d^{4} l}{(2 \pi)^{4}} \frac{e^{-i \tilde{p} l-\frac{i}{2} \sum_{j<k} \tilde{p}_{j} p_{k} \nu_{k}}}{l^{2}\left(l+p_{1}\right)^{2} \ldots\left(l+p_{1}+. .+p_{N-1}\right)^{2}} .
$$

The integral appearing in this expression coincides with that of the N-point function of a non-commutative $\Phi^{3}$ theory and has been calculated in [30, 31] (see also [32 for a recent two-loops analysis). There, a small mass $m$ for the field $\Phi$ was introduced as an ordinary infrared regulator. The evaluation of the previous integral gives 1

$$
J_{* n}(-p)\left(\frac{\tilde{p}}{m}\right)^{N-2} K_{N-2}(m \tilde{p}) J_{* N-n}(p)
$$

where $K_{N-2}$ are modified Bessel functions. We have denoted by $J_{* n}(-p)$ the kernel of the $*_{n}$-product defined in [33], i.e. $J_{* n}(-p) \equiv J\left(-p_{r(1)}, . .,-p_{r(n)}\right)$ where $p_{r(j)}$ are the $n$ momenta entering the twisted vertices and $p=\sum p_{r(j)}$. A comment is now in order. Expression (28) is not the complete answer, but the leading term in the infrared. Subleading terms are suppressed by powers of $\tilde{p}^{2} p_{i} p_{j}$ and therefore they do not give rise to infrared divergences for any $N$ f. In the following, we will keep in the evaluation of the N-point functions only the infrared-leading term. Then, (27) reduces to

$$
\frac{1}{2 \pi^{2}}(-i g)^{N} \sum_{\nu_{i}}(-)^{n} J_{* n}(-p)\left[\partial_{\mu_{1}} \ldots \partial_{\mu_{N}}\left(\frac{\tilde{p}}{m}\right)^{N-2} K_{N-2}(m \tilde{p})\right] J_{* N-n}(p) .
$$

Using the properties of the modified Bessel functions, it is easy to see that the term in square brackets gives rise to a contribution of the form

$$
(-)^{N} \tilde{p}^{\mu_{1}} \ldots \tilde{p}^{\mu_{N}} \frac{m^{2}}{\tilde{p}^{2}} K_{2}(m \tilde{p}) \text {. }
$$

\footnotetext{
${ }^{1}$ This result is not affected by considering $p$ and $\tilde{p}$ as independent variables.

${ }^{2}$ It is interesting that the subleading terms do not seem to have such a simple expression in terms of $*_{n}$ products as (28).
} 
Adding up all such contributions to the effective action we get

$$
\begin{aligned}
S_{e f f}^{I}= & \frac{1}{2 \pi^{2}} \sum_{N=2}^{\infty}(i g)^{N} \int d^{4} p \sum_{n=1}^{N-1} \frac{(-)^{n}}{n !(N-n) !} \\
& \frac{m^{2}}{\tilde{p}^{2}} K_{2}(m \tilde{p}) \tilde{p}^{\mu_{1}} \ldots \tilde{p}^{\mu_{N}}\left[A_{\mu_{1}} . . A_{\mu_{n}}\right]_{* n}(-p)\left[A_{\mu_{n+1}} . . A_{\mu_{N}}\right]_{* N-n}(p) .
\end{aligned}
$$

This expression reproduces (23) by setting $2 f(\tilde{p})=\frac{m^{2}}{\tilde{p}^{2}} K_{2}(m \tilde{p})$. Although the $*_{n}$ also appear in the effective action of the non-commutative $\Phi^{3}$ theory, they only combine to form the scalar analog of Wilson loop operators in the limit of large non-commutative parameter [31]. On the contrary, the invariance of the effective action with respect to gauge transformations of the background field suggests that, in gauge theories, Wilson loop operators will play an important role for any value of $\theta$. As a first example, a Wilson loop completion of the non-planar contributions to the $F^{4}$ terms in $\mathcal{N}=4$ gauge theory has been proposed in [34]. We have just seen that the puzzling polelike divergent terms originating from UV/IR mixing are part of the simplest gauge-invariant double-trace operator that can appear in the effective action. We will leave for the next section the extension of the previous considerations to gauge theories with adjoint matter.

\subsection{The Logarithms}

We would like to comment on the IR logarithmic-divergent terms arising from UV/IR mixing. As already mentioned, these subleading contributions occur also in the supersymmetric case. We suggest here a gauge invariant completion of the IR logarithmic divergent terms. This suggestion is not as rigorous as the derivation in the previous subsection, but our result is fixed by the requirement of gauge invariance.

It was shown in 18 that the logarithmic singularities of the 2-, 3- and 4-point function of pure NC $U(1)$, in the limit $\left|\tilde{p}_{i}\right| \sim\left|\tilde{p}_{i}+\tilde{p}_{j}\right| \sim \theta \Lambda_{I R} \rightarrow 0$, combine into the following contribution to the effective action:

$$
S_{e f f}^{I I}=\frac{1}{4} \beta_{0} \log \left(\theta \Lambda_{\mathrm{IR}}\right)^{2} \int \mathrm{d}^{4} \mathrm{xF}^{\mu \nu} \mathrm{F}_{\mu \nu},
$$

with $\Lambda_{I R}$ an infrared cut-off and $\beta_{0}$ the coefficient of the 1-loop beta function. It is tempting to propose the following gauge-invariant completion of (32), 
which generalizes to the $U(N)$ case

$$
S_{\text {eff }}^{I I}=\frac{1}{4} \beta_{0} \int d^{4} p \mathcal{O}^{\mu \nu}(-p) K_{0}(m \tilde{p}) \mathcal{O}_{\mu \nu}(p),
$$

where the operator $\mathcal{O}_{\mu \nu}$ is defined by

$$
\mathcal{O}_{\mu \nu}(p)=\operatorname{tr} \int \mathrm{d}^{4} \mathrm{x} \mathrm{L}_{*}\left(\mathrm{~F}_{\mu \nu}(\mathrm{x}) \mathrm{e}^{\mathrm{ig} \int_{0}^{1} \mathrm{~d} \sigma \tilde{\mathrm{p}}^{\mu} \mathrm{A}_{\mu}(\mathrm{x}+\tilde{\mathrm{p}} \sigma)}\right) * \mathrm{e}^{\mathrm{ipx}} .
$$

Following the notation of [34, $L_{*}$ denotes integration of $F_{\mu \nu}$ along the open Wilson line together with path ordering with respect of the $*$-product of all terms inside the parenthesis. The action (33) reproduces the pure gluonic log-like N-point functions (21) in the small $m$ limit.

\section{The Effective Action via Closed Strings Exchange}

The recent interest in the study of non-commutative field theories has been mainly motivated by their connection to string theory. The world-volume coordinates of D-branes in the presence of a constant B-field background turn out to satisfy the relation $\left[x^{\mu}, x^{\nu}\right]=i \theta^{\mu \nu}$, with $\theta^{\mu \nu} \sim 1 / B_{\mu \nu}$. As a consequence, the low energy theory on the brane is a non-commutative gauge theory. In this section we would like to analyze (23) and (33) (or (15) and (21)) from a string-inspired point of view. In a series of recent papers it has been shown that closed string modes couple to non-commutative D-branes through open Wilson line operators [34, 35, 36, 37]. This result was obtained by evaluating the disk amplitude between a closed string and open string modes.

Let us consider the annulus diagram with boundaries on non-commutative D-branes as in fig.2. It can be seen as a loop of open strings or a tree level exchange of closed strings. In the limit of a large cylinder the closed string channel picture is more adequate since the annulus diagram factorizes to closed string insertions on a disk connected by a closed string propagator [38]. In the opposite limit of a small cylinder, the exchange of the lowest open string modes dominates. This provides the field theory limit, and the annulus amplitude reproduces the 1-loop field theory effective action. Thus in 


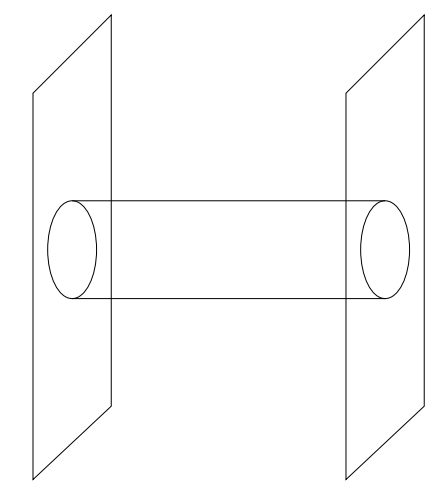

Figure 2: The annulus amplitude.

general we could expect in the field theory effective action more complicated contributions than (23) and (33), which structurally are reminiscent of a closed string exchange. Notice that a similar structure was proposed as the gauge-invariant completion of the non-planar $F^{4}$ terms in the effective action of $\mathcal{N}=4$ non-commutative Yang-Mills [33, 34]. In that case, the function $f$ had the interpretation of a closed string propagator in type II string theory. This however comes as no surprise since the $F^{4}$ terms in the maximally supersymmetric case are protected by non-renormalization theorems 38. In contrast, it is remarkable that (23) emerges in a non-supersymmetric theory. We will show below that the function $f$ appearing in the IR-divergent terms can also be directly related to a closed string propagator.

In the rest of this section we will consider type 0 string theory. This theory can be obtained as a world-sheet orbifold of type II, which projects out space-time fermions. It contains a closed string tachyon arising from the twisted sectors. There are however no open string tachyons on D-branes in type 0 theory. This makes it especially adequate for our considerations. We will work with the gauge theory on $N$ electric D3-branes. It is given by the dimensional reduction of pure Yang-Mills in 10 dimensions, i.e. gauge fields plus 6 scalars in the adjoint representation.

We start by analysing which closed string modes couple to the open Wilson line operator (22). The first candidate is the type 0 tachyon. In the absence of B-field and at leading order in $\alpha^{\prime}$, it couples to the brane tension 
as 39, 40,

$$
\frac{N}{4\left(2 \pi \alpha^{\prime}\right)^{2}}
$$

Following the same analysis done for bosonic and type II string theory [34, [36], it is easy to see that the trivial field theory operator (35) gets promoted to an open Wilson loop in the presence of a B-field. The coupling of the type 0 tachyon to the D-brane field theory at leading $\alpha^{\prime}$ order is described by

$$
S^{I}=\frac{\kappa_{10}}{g_{Y M}^{2}} \int \frac{d^{10} P}{(2 \pi)^{10}} \sqrt{\operatorname{det} \mathrm{G}} T(P) \mathcal{O}(-P),
$$

where $G$ is the open string metric and

$$
\mathcal{O}(P)=\frac{1}{4\left(2 \pi \alpha^{\prime}\right)^{2}} \operatorname{tr} \int d^{4} x W(x, C) * e^{i p x} .
$$

We denote by $P_{M}$ the 10-dimensional momentum, $p_{\mu}$ the momentum along the 4-dimensional world-volume of the D3-brane and $p_{\perp_{i}}$ the momentum in the transverse directions. In the previous expressions $W(x, C)$ is a generalization of (22) which involves the transverse scalars

$$
W(x, C)=P_{*}\left(e^{i g \int_{0}^{1} d \sigma \tilde{p}^{\mu} A_{\mu}(x+\tilde{p} \sigma)+y_{i} \phi^{i}(x+\tilde{p} \sigma)}\right),
$$

where we have defined $y_{i}=2 \pi \alpha^{\prime} p_{\perp_{i}}$ and $\phi^{i}=X^{i} / 2 \pi \alpha^{\prime}$ for $i=1, \ldots, 6$, which provides the correct normalization for the field theory scalar fields.

The on-shell condition for the type 0 tachyon is $P_{M} g^{M N} P_{N}=-2 / \alpha^{\prime}$, with $g$ the closed string metric. Closed and open string metrics are related by $g^{-1}=G^{-1}-\theta G \theta /\left(2 \pi \alpha^{\prime}\right)^{2}$ [2]. In the Seiberg-Witten limit, i.e. $\alpha^{\prime} \rightarrow 0$ keeping $G$ and $\theta$ fixed, the on-shell condition becomes [34, 36]

$$
\tilde{p}^{2}+y^{2}=8 \pi^{2} \alpha^{\prime} .
$$

The closed string mass is a subleading effect with respect to the momentum in the non-commutative directions in the Seiberg-Witten limit. In spite of that, it will be crucial in the following to keep its contribution to the massshell condition. We want to analyse how the tachyon exchange contributes to the annulus amplitude. For two D3-branes separated by a distance $r$ we obtain

$$
S_{e f f}^{I}=\frac{\kappa_{10}^{2}}{g_{Y M}^{4}} \int \frac{d^{10} P}{(2 \pi)^{10}} \frac{\operatorname{det} G}{\sqrt{\operatorname{det} g}} \mathcal{O}(P) \mathcal{O}(-P) \frac{e^{i p_{\perp} r}}{\frac{M^{2}}{\left(2 \pi \alpha^{\prime}\right)^{2}}+p_{\perp}^{2}}
$$


The quantity $M^{2} /\left(2 \pi \alpha^{\prime}\right)^{2}$ is the effective mass of the closed string tachyon propagating in the six transverse dimensions; from (39) $M^{2}=\tilde{p}^{2}-8 \pi^{2} \alpha^{\prime}$.

In order to make contact with the previous section we will first consider the dependence of $\mathcal{O}$ on the gauge fields only. Then $\mathcal{O}=\frac{1}{\left(4 \pi \alpha^{\prime}\right)^{2}} W(p)$, with $W(p)$ given by (22). Using (see for example [34])

$$
\frac{\kappa_{10}^{2}}{g_{Y M}^{4}}=\pi\left(2 \pi \alpha^{\prime}\right)^{4} \frac{\sqrt{\operatorname{det} g}}{\sqrt{\operatorname{det} G}}
$$

and defining $m=r / 2 \pi \alpha^{\prime}$, the previous expression can be rewritten as

$$
S_{e f f}^{I}=\frac{\pi}{\left(4 \pi \alpha^{\prime}\right)^{4}} \int \frac{d^{4} p}{(2 \pi)^{4}} \sqrt{\operatorname{det} G} \operatorname{tr} W(p) \operatorname{tr} W(-p) G(p),
$$

where

$$
G(p)=\int \frac{d^{6} y}{(2 \pi)^{6}} \frac{e^{i y m}}{M^{2}+y^{2}}=\frac{1}{(2 \pi)^{3}} \frac{M^{2}}{m^{2}} K_{2}(m M) .
$$

$G(p)$ represents the closed string propagator in the transverse dimensions, rescaled appropriately to the field theory limit. Indeed, it is finite in the limit $\alpha^{\prime} \rightarrow 0$. However (42) diverges in this limit due to the $O\left(\alpha^{\prime-2}\right)$ dependence of the brane tension to which the tachyon couples. We can define a finite contribution to (42) by expanding $G(p)$ to $O\left(\alpha^{\prime 4}\right)$, using the explicit dependence of $M^{2}$ on $\alpha^{\prime}$. We then obtain a contribution to the field theory effective action of the form (23), with

$$
\left.f(p) \equiv G(p)\right|_{\alpha^{\prime 4}}=c \frac{m^{2}}{\tilde{p}^{2}} K_{2}(m \tilde{p}),
$$

with $c=\frac{4 \pi^{5}}{3}$. This agrees with the result derived from the field theory calculation, up to a global coeficient. We will comment on this below.

Notice that in order to obtain the IR divergent terms from the string exchange, it was essential that the field theory operator that couples to the tachyon carries negative powers of $\alpha^{\prime}$. The reason for this is that at $\mathcal{O}\left(\alpha^{\prime 0}\right)$, $G \sim 1 / m^{4}$ as $\tilde{p} \rightarrow 0$. Such a term is related to the ordinary infrared problems of a field theory with massless degrees of freedom. However, remarkably, $G(p)$ contains information about the new divergences due to UV/IR mixing effects in non-commutative field theories when expanded to higher orders in $\alpha^{\prime}$. We have analyzed above the coupling of the type 0 tachyon to the Dbrane field theory at leading order in $\alpha^{\prime}$. At $\mathcal{O}\left(\alpha^{\prime 0}\right)$ it couples to the field 
theory operators $\operatorname{tr} F^{2}$ and $\left(D \phi^{i}\right)^{2}$ [39, 40]. For the reasons just exposed, the coupling of the tachyon to these operators would contribute non-singular terms in the effective action and therefore we will not consider them.

Equation (42) differs from (23), as we subtracted the '1' from the open Wilson line in (23). The ' 1 ' in coordinate space is in fact $\delta(p)$, as we work in Fourier space, and therefore this difference affects only the $p_{\mu}=0$ component of $W$. We would like to stress that the expansion of $G(p)$ in $\alpha^{\prime}$ powers requires that $\tilde{p}$ is non zero. At $\tilde{p}=0$ and in the limit $\alpha^{\prime} \rightarrow 0$, the string propagator is $G \sim 1 / m^{4}$. The associated contribution to the effective action is proportional to

$$
\frac{1}{\left(\alpha^{\prime} m\right)^{4}} \delta^{(4)}(0) \rightarrow \Lambda^{4} \int d^{4} x
$$

where $1 /\left(\alpha^{\prime} m\right) \sim \Lambda$ can be interpreted as a field theory scale. Therefore the difference between (42) and (23) reflects the vacuum energy of the gauge theory, which is taken into account in the string theory calculation. Once this infinity is substracted, the string exchange just reproduces the field theory result (23).

We will now show that (40) can also reproduce the pole-like divergent terms associated with the adjoint scalars. Expanding $\mathcal{O}$ to linear order in the fields, we obtain the following contribution involving the adjoint scalars

$$
S_{e f f}^{\prime I} \sim \int \frac{d^{4} p}{(2 \pi)^{4}} \sqrt{\operatorname{det} G} \operatorname{tr} \phi_{i}(p) \operatorname{tr} \phi_{j}(-p) f_{i j}(p)
$$

where

$$
\begin{aligned}
f_{i j}= & \left.\int \frac{d^{6} y}{(2 \pi)^{6}} \frac{e^{i y m} y_{i} y_{j}}{M^{2}+y^{2}}\right|_{\alpha^{\prime 4}}= \\
= & -\partial_{m_{i}} \partial_{m_{j}} G(p)=c\left(\delta_{i j} \frac{m}{\tilde{p}} K_{1}(m \tilde{p})+m_{i} m_{j} K_{0}(m \tilde{p})\right) .
\end{aligned}
$$

The first term in (47) leads to the action

$$
S_{e f f}^{\prime I} \sim \int \frac{d^{4} p}{(2 \pi)^{4}} \sqrt{\operatorname{det} G} \operatorname{tr} \phi_{i}(p) \operatorname{tr} \phi_{i}(-p) \frac{m}{\tilde{p}} K_{1}(m \tilde{p}),
$$

which corresponds, in the $m \tilde{p} \rightarrow 0$ limit, to the pole-like contribution in the effective action of the scalars (15). The second term in (47) yields a $m^{2} \log$ 
contribution which vanishes when $m \rightarrow 0$. Notice that while $f$ in (44) tends to $2 c / \tilde{p}^{4}$ in the infrared limit, $f_{i j}$ tends to $c / \tilde{p}^{2}$. This reproduces the relative factor of two between the pole-like contributions to the propagator of the gauge field and adjoint scalars, eq.(9),(10). The same applies to the linear poles of the 3-point functions. Therefore the gauge invariant expression (42), defined such that we only keep the finite terms in the $\alpha^{\prime} \rightarrow 0$ limit, accounts for all pole-like divergent terms of the field theory up to a global coefficient.

The discrepancy in the global coefficient can be related to the fact that not only the tachyon, but also massive scalar closed strings couple to the brane tension. In the Seiberg-Witten limit these contributions are of the same form as that of the tachyon, since momentum in the non-commutative directions dominates over the oscillator mass. Thus they will renormalize the overall coefficient in front of the effective action. To summarize, we have seen that the gauge invariant effective action containing the infrared poles can be directly related to a closed string exchange between D-branes. It is tempting to think of this as the exchange of an "effective closed string mode". Remarkably, among the original closed string modes that contribute to this effect is the tachyon mode.

We will briefly address the log like contributions which appear also in the supersymmetric field theory (21). Consider a two-form (denoted by $M_{M N}$ ) closed string which couples to the operator $\mathcal{O}^{M N}$ (separated into $4 \mathrm{~d}$ and $6 \mathrm{~d}$ indices):

$$
S^{I I}=\frac{\kappa_{10}}{g_{Y M}^{2}} \int \frac{d^{10} P}{(2 \pi)^{10}} \sqrt{\operatorname{det} \mathrm{G}}\left(M_{\mu \nu}(P) \mathcal{O}^{\mu \nu}(-P)+M_{\mu i}(P) \mathcal{O}^{\mu i}(-P)\right),
$$

with

$$
\begin{aligned}
& \mathcal{O}^{\mu \nu}(P)=\frac{1}{2 \pi \alpha^{\prime}} \operatorname{tr} \int d^{4} x L_{*}\left(F^{\mu \nu} W(x, C)\right) * e^{i p x} \\
& \mathcal{O}^{\mu i}(P)=\frac{1}{2 \pi \alpha^{\prime}} \operatorname{tr} \int d^{4} x L_{*}\left(D^{\mu} \phi^{i} W(x, C)\right) * e^{i p x} .
\end{aligned}
$$

Repeating the same steps as for the tachyon field we can write the effective action due to an exchange of a massive 2 -form as

$$
S_{e f f}^{I I} \sim \int \frac{d^{4} p d^{6} y}{(2 \pi)^{10}} \sqrt{\operatorname{det} G} \mathcal{O}^{M N}(p, y) \mathcal{O}_{M N}(-p,-y) \frac{e^{i y m}}{M^{2}+y^{2}}
$$


with $M^{2}=\tilde{p}^{2}+8 \pi^{2} l \alpha^{\prime}$ and $l$ some positive integer number which corresponds to the string excitation number. For simplicity let us set the adjoint scalar fields to zero in $W(x, C)$, which does not affect gauge invariance. We get then

$$
S_{e f f}^{I I} \sim \int \frac{d^{4} p}{(2 \pi)^{4}}\left(\mathcal{O}_{\mu \nu}(p) \mathcal{O}^{\mu \nu}(-p)+\mathcal{O}_{\mu i}(p) \mathcal{O}^{\mu i}(-p)\right) G(p)
$$

but now we should simply keep the terms in $G(p)$ which are $O\left(\alpha^{\prime 2}\right)$. This yields

$$
\left.G(p)\right|_{\alpha^{\prime 2}} \sim K_{0}(m \tilde{p}) .
$$

which reproduces the action (33) and in addition the log like pieces of the scalars (21).

Thus, we have shown that the logarithmic like ( $K_{0}$, in fact) contribution to effective action can be understood from massive 2-form closed strings exchange. Note that the massless NS-NS 2 form does not contribute here. Only massive modes. Another comment is that we could not reproduce the overall factor in front of the effective action, $\beta_{0}$. The understanding of the overall factor, from the string theory point of view, is equivalent to the understanding of the weight of each individual massive string in the coupling to the operator $F^{M N}$ on the brane. We will not address this problem here.

\section{Discussion}

In this work we have discussed UV/IR effects in a non-supersymmetric gauge theory. Our main results are the effective actions (2) and (3). These actions incorporate the two kinds of non-planar singular amplitudes: the poles and the logs.

The log like contributions exist also in the supersymmetric theory, apart from the $\mathcal{N}=4$ SYM theory. The picture that emerges from our work is that one can understand these effects as due to an exchange of massive two-form closed strings which couple to the operator $\operatorname{tr} F_{\mu \nu}$.

The more interesting contributions are the poles. These poles cancel in the supersymmetric gauge theory. Our picture here is that these terms can be understood as due to an exchange of a tachyon and massive scalar closed strings that couple to the brane tension. In the superstring theory there is no tachyon. Moreover, the contributions from the NS-NS sector cancel the 
contributions from the R-R sector and this is our explanation of why we do not see such effects in the (super-)gauge theory side.

The (partial) contribution of the closed string tachyon to the tachyonic instabilities of the non-commutative theory suggests that the two phenomena are related. Indeed, it is true that the poles are also due to massive closed strings, since in the Seiberg-Witten limit all the massive tower contributes similarly to the exchange between the D-branes [8]. Therefore we do not argue that the tachyon in the field theory has a one to one correspondence with the closed string tachyon. The relation is more indirect. We have not found any example of a non-supersymmetric string theory with a NS-NS two form which does not contain a closed string tachyon (or a tree level open string tachyon). It is possible to construct a non-tachyonic nonsupersymmetric string theory [41, 42] by using a special orientifold, but the orientifold removes the NS-NS two form from the spectrum. In addition, in non-supersymmetric string theories, such as strings on orbifold singularities, there is always a tachyon in the twisted sector. In these cases the non-commutative field theory on the brane is 'tachyonic'. Namely, in all these constructions there are more bosons in the adjoint representation than

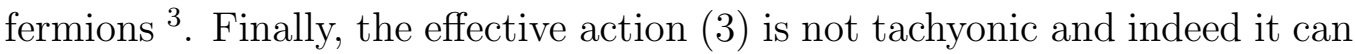
be understood as due to massive 2-forms exchange (no closed string tachyon is involved in this case). These observations support our claim about a relation between the closed string tachyon and the generated tachyon on the brane. We suggest that the closed string tachyon that couples non-trivially to the brane (in contrast to the commutative theory), is behind the instabilities in the field theory. This point of view is somewhat similar in spirit to [43], where it is was argued that a field theory which is holographically dual to a tachyonic string theory should suffer from instabilities.

In the light of our picture, we would like to address the problem of the stability of the non-commutative non-supersymmetric Yang-Mills theory. Since this theory is tachyonic, similarly to type 0 string theory, we suggest that the consistency issue is related to the fate of the closed string tachyon. Type 0 string theory might be consistent if tachyon condensation occurs (for concrete suggestions see [44, 45, 46]). In particular, the true vacuum of the type 0 string might be supersymmetric ! It is tempting to suggest that if this is the case, there will be examples of non-commutative non-supersymmetric gauge

\footnotetext{
${ }^{3}$ A.A. would like to thank Rodolfo Russo for discussions on this issue.
} 
theories which are consistent and that (1) is a consequence of the expansion around the perturbative vacuum.

\section{ACKNOWLEDGEMENTS}

We would like to thank Luis Alvarez-Gaume, Carlo Angelantonj, Jose Barbon, Chong-Sun Chu, Yaron Oz and Rodolfo Russo for discussions. Part of the work of A.A. was done at the Theoretical Physics Centre of the Ecole Polytechnique. A.A. wishes to take this opportunity to thank all members of the String Theory Group there.

\section{References}

[1] A. Connes, M. R. Douglas and A. Schwarz, "Noncommutative geometry and matrix theory: Compactification on tori," JHEP 9802, 003 (1998) [hep-th/9711162].

[2] N. Seiberg and E. Witten, "String theory and noncommutative geometry," JHEP 9909, 032 (1999) [hep-th/9908142].

[3] A. Gonzalez-Arroyo and M. Okawa, "A Twisted Model For Large N Lattice Gauge Theory," Phys. Lett. B 120, 174 (1983).

[4] A. Gonzalez-Arroyo and M. Okawa, "The Twisted Eguchi-Kawai Model: A Reduced Model For Large N Lattice Gauge Theory," Phys. Rev. D 27, 2397 (1983).

[5] T. Filk, "Divergencies in a field theory on quantum space," Phys. Lett. B 376 (1996) 53.

[6] S. Minwalla, M. Van Raamsdonk and N. Seiberg, "Noncommutative perturbative dynamics," JHEP 0002, 020 (2000) hep-th/9912072.

[7] A. Matusis, L. Susskind and N. Toumbas, "The IR/UV connection in the non-commutative gauge theories," JHEP 0012, 002 (2000) hepth/0002075]. 
[8] G. Arcioni, J. L. Barbon, J. Gomis and M. A. Vazquez-Mozo, "On the stringy nature of winding modes in noncommutative thermal field theories," JHEP 0006, 038 (2000) hep-th/0004080.

[9] I. Y. Arefeva, D. M. Belov, A. S. Koshelev and O. A. Rychkov, "Renormalizability and UV/IR mixing in noncommutative theories with scalar fields," Phys. Lett. B 487, 357 (2000).

[10] A. Micu and M. M. Sheikh Jabbari, "Noncommutative $\phi^{4}$ theory at two loops," JHEP 0101, 025 (2001).

[11] I. Chepelev and R. Roiban, "Convergence theorem for non-commutative Feynman graphs and renormalization," JHEP 0103, 001 (2001) hepth/0008090].

[12] L. Griguolo and M. Pietroni, "Wilsonian renormalization group and the non-commutative IR/UV connection," JHEP 0105, 032 (2001) hepth/0104217].

[13] Y. Kinar, G. Lifschytz and J. Sonnenschein, "UV/IR connection: A matrix perspective," JHEP 0108, 001 (2001) hep-th/0105089.

[14] M. Hayakawa, "Perturbative analysis on infrared and ultraviolet aspects of noncommutative QED on $R^{4}$," hep-th/9912167.

[15] A. Bilal, C. Chu and R. Russo, "String theory and noncommutative field theories at one loop," Nucl. Phys. B 582, 65 (2000) hep-th/0003180.

[16] A. Armoni, "Comments on perturbative dynamics of non-commutative Yang-Mills theory," Nucl. Phys. B 593, 229 (2001) hep-th/0005208].

[17] K. Landsteiner, E. Lopez and M. H. Tytgat, "Excitations in hot noncommutative theories," JHEP 0009, 027 (2000) [hep-th/0006210].

[18] C. P. Martin and F. Ruiz Ruiz, "Paramagnetic dominance, the sign of the beta function and UV/IR mixing in non-commutative U(1)," Nucl. Phys. B 597, 197 (2001) hep-th/0007131.

[19] M. Pernici, A. Santambrogio and D. Zanon, "The one-loop effective action of noncommutative $\mathrm{N}=4$ super Yang-Mills is gauge invariant," Phys. Lett. B 504, 131 (2001) hep-th/0011140. 
[20] V. V. Khoze and G. Travaglini, "Wilsonian effective actions and the IR/UV mixing in noncommutative gauge theories," JHEP 0101, 026 (2001) hep-th/0011218.

[21] D. Zanon, "Noncommutative $\mathrm{N}=1,2$ super $\mathrm{U}(\mathrm{N})$ Yang-Mills: UV/IR mixing and effective action results at one loop," Phys. Lett. B 502, 265 (2001) hep-th/0012009.

[22] F. R. Ruiz, "Gauge-fixing independence of IR divergences in noncommutative $\mathrm{U}(1)$, perturbative tachyonic instabilities and supersymmetry," Phys. Lett. B 502, 274 (2001) hep-th/0012171.

[23] K. Landsteiner, E. Lopez and M. H. Tytgat, "Instability of noncommutative SYM theories at finite temperature," JHEP 0106, 055 (2001) hep-th/0104133.

[24] A. Armoni and R. Russo, "Non-commutative gauge theories and the cosmological constant," hep-th/0106189.

[25] M. R. Douglas and N. A. Nekrasov, "Noncommutative field theory," hep-th/0106048.

[26] R. J. Szabo, "Quantum Field Theory on Noncommutative Spaces," hepth/0109162.

[27] N. Ishibashi, S. Iso, H. Kawai and Y. Kitazawa, Nucl. Phys. B 573, 573 (2000) hep-th/9910004.

[28] D. J. Gross, A. Hashimoto and N. Itzhaki, "Observables of noncommutative gauge theories," hep-th/0008075.

[29] M. Van Raamsdonk, "The Meaning of Infrared Singularities in Noncommutative Gauge Theories," hep-th/0110093.

[30] Y. Kiem, S. J. Rey, H. T. Sato and J. T. Yee, "Open Wilson lines and generalized star product in nocommutative scalar field theories," hepth/0106121.

[31] Y. Kiem, S. Rey, H. Sato and J. Yee, "Anatomy of one-loop effective action in noncommutative scalar field theories," hep-th/0107106. 
[32] Y. Kiem, S. S. Kim, S. J. Rey and H. T. Sato, "Anatomy of Two-Loop Effective Action in Noncommutative Field Theories," hep-th/0110066.

[33] H. Liu and J. Michelson, "*-Trek: The one-loop $\mathrm{N}=4$ noncommutative SYM action," hep-th/0008205.

[34] H. Liu, “*-Trek II: *n operations, open Wilson lines and the SeibergWitten map," hep-th/0011125.

[35] S. R. Das and S. P. Trivedi, "Supergravity couplings to noncommutative branes, open Wilson lines and generalized star products," JHEP 0102, 046 (2001) hep-th/0011131.

[36] Y. Okawa and H. Ooguri, "How noncommutative gauge theories couple to gravity," Nucl. Phys. B 599, 55 (2001) hep-th/0012218.

[37] H. Liu and J. Michelson, "Supergravity couplings of noncommutative D-branes," hep-th/0101016.

[38] M. R. Douglas, D. Kabat, P. Pouliot and S. H. Shenker, "D-branes and short distances in string theory," Nucl. Phys. B 485, 85 (1997) thepth/9608024].

[39] I. R. Klebanov and A. A. Tseytlin, "Asymptotic freedom and infrared behavior in the type 0 string approach to gauge theory," Nucl. Phys. B 547, 143 (1999) hep-th/9812089.

[40] M. R. Garousi, "String scattering from D-branes in type 0 theories," Nucl. Phys. B 550, 225 (1999) hep-th/9901085.

[41] A. Sagnotti, "Some properties of open string theories," hep-th/9509080.

[42] A. Sagnotti, "Surprises in open-string perturbation theory," Nucl. Phys. Proc. Suppl. 56B, 332 (1997) hep-th/9702093.

[43] I. R. Klebanov, "Tachyon stabilization in the AdS/CFT correspondence," Phys. Lett. B 466, 166 (1999) [hep-th/9906220.

[44] O. Bergman and M. R. Gaberdiel, "Dualities of type 0 strings," JHEP 9907, 022 (1999) hep-th/9906055. 
[45] M. S. Costa and M. Gutperle, "The Kaluza-Klein Melvin solution in M-theory," JHEP 0103, 027 (2001) hep-th/0012072.

[46] A. Adams, J. Polchinski and E. Silverstein, "Don't panic! Closed string tachyons in ALE space-times," hep-th/0108075. 